\title{
Design, Implementation and Testing of a Real-Time Mobile WiMAX Testbed Featuring MIMO Technology
}

\author{
Oriol Font-Bach ${ }^{1}$, Nikolaos Bartzoudis ${ }^{1}$, Antonio Pascual-Iserte ${ }^{1,2}$ \\ and David López Bueno ${ }^{1}$ \\ ${ }^{1}$ Centre Tecnològic de Telecomunicacions de Catalunya (CTTC), Parc Mediterrani de la \\ Tecnologia (PMT), Av. Carl Friedrich Gauss 7, 08860 Castelldefels, Barcelona, Spain \\ ${ }^{2}$ Dept. of Signal Theory and Communications - Universitat Politècnica de Catalunya (UPC), \\ Campus Nord, Jordi Girona 1-3, 08034 Barcelona, Spain \\ \{ofont,nbartzoudis,dlopez\}@cttc.cat - antonio.pascual@upc.edu
}

\begin{abstract}
Multiple input multiple output (MIMO) is a technology that enhances wireless systems capacity, data rate, and coverage by utilizing the spatial diversity provided by multiple antennas. However, these benefits come at the expense of increased computational complexity. Implementing a broadband MIMO wireless communication system in a real-time testbed is a challenging task, entailing numerous pitfalls. This paper presents several implementation aspects of a real-time MIMO testbed based on the mobile WiMAX standard. The focus is mainly laid on the bit-intensive baseband digital signal processing at the receiver.
\end{abstract}

Keywords: MIMO, testbeds, IEEE 802.16e, real-time systems, FPGAs, DSP.

\section{Introduction}

Deploying a broadband wireless communication standard such as the IEEE 802.16e-2005 [1] (i.e., mobile WiMAX) in a real-time testbed implies several design, implementation and testing challenges, especially considering the top-up computational complexity introduced by the MIMO technology. The massive parallelism required for the baseband signal processing in real-time testbeds, makes the FPGA devices the obvious candidate for implementing such systems. The inherent processing parallelism of FPGA devices and the availability of a wide range of preverified IP-cores make them a preferable choice compared to DSP microprocessors. At the same time, the cell-processors though demonstrating a remarkable performance [2] are still considered to be an immature solution due to the C-coding parallelism limitations, the insufficient IP libraries and the lack of development boards.

This paper presents the challenging and demanding task of implementing a pointto-point mobile WiMAX system in a real-time testbed having a $2 \times 2$ MIMO configuration. The system uses matrix A encoding in an open-loop configuration (i.e. without feedback), based on Alamouti's space-time block code [3] in a per carrier basis. The $20 \mathrm{MHz}$ channel bandwidth of this testbed exceeds the WiMAX Forum specifications for the IEEE 802.16e-2005 standard (i.e. wave-2) positioning the

This work was partially supported by the Catalan Government under grant 2009 SGR 891; by the Spanish Government under projects TEC2008-06327-C03 (MULTI-ADAPTIVE) and 2A103 (MIMOWA) from MEDEA+ program (AVANZA I+D TSI-020400-2009-44) and "Torres Quevedo" grants PTQ-08-01-06441, PTQ06-02-0540, PTQ06-2-0553; and by the European Commission under projects NEWCOM++ (216715) and BuNGee (248267). 
system presented herein on the forefront of applied research utilizing real-time MIMO testbeds.

Setting up the whole testbed is a quite hard research and engineering task. The most critical part of the mentioned development is found in the design, simulation, implementation and real-time debugging of the receiver which, for this reason, is widely detailed in this paper.

\section{Short Review of MIMO Testbeds}

The great majority of the existing testbeds supports off-line signal processing, making use of Matlab or other signal processing software [4], [5]. Apparently, off-line testbeds are not able to process in real-time the received signals. However, their flexibility makes them appealing to researchers since such testbeds allow them to explore various real-world signal processing concepts. Their offline operation renders these testbeds incapable to explore medium access control protocols and the reception of long data frames because of timing and memory constraints, respectively. Besides, offline testbeds are not able to realize closed-loop strategies.

Although we have found in the literature low-bandwidth MIMO testbeds based on the IEEE $802.11 \mathrm{n}$ [6] and the $802.16 \mathrm{~d}$ standard [7], [8] (i.e. no mobility), we have not encountered literature for real-time MIMO testbeds implementing the IEEE 802.16e standard using a $20 \mathrm{MHz}$ bandwidth. A combination of mobile and fixed WiMAX testbed is presented in [9]; nevertheless the scope of the project is different since commercial equipment is used to assemble the entire physical layer of the testbed. Real-time MIMO testbeds implementing the IEEE 802.16e-2005 standard are mainly deployed by industrial initiatives (e.g. Alvarion), which are currently offering bandwidths up to $10 \mathrm{MHz}$ (e.g. WiMAX wave-2 specifications [1]).

\section{Description of the Experimental Setup}

A point-to-point MIMO testbed typically comprises i) a transmitter with baseband signal processing units, digital-to-analog converters (DACs) and RF up-converters, ii) a multi channel emulator or sets of transmit and receive antennas (indoor channel), and iii) a receiver with a series of RF down-converters, analog-to-digital converters (ADCs) and baseband digital signal processing units. A graphic-overview of our realtime mobile WiMAX testbed setup for a point-to-point $2 \times 2$ MIMO system is shown in figure 1. The parameters of the OFDM downlink (DL) frame, consisting of a single burst with a fixed predefined format (i.e, FCH and DL-MAP are not decoded), are shown in table 1 and depicted in figure 2 .

Taking into account the baseband sampling frequency (i.e. $22.4 \mathrm{MHz}$ ), the rate of Alamouti's space-time code (STC) coding (i.e. unity) and the total number of PUSC subcarriers (i.e. data + pilot + dc-carrier $=1681$ ), the actual peak of un-coded useful data rate is $22.4 \times 2 \times 1 \times 1681 / 2048 \times 2048 / 2560=29.4175 \mathrm{Mbits} / \mathrm{s}$ and the spectrum efficiency for a $20 \mathrm{MHz}$ channel bandwidth is $1.47 \mathrm{bits} / \mathrm{Hz} / \mathrm{s}$ assuming the use of QPSK modulation at each data carrier. 


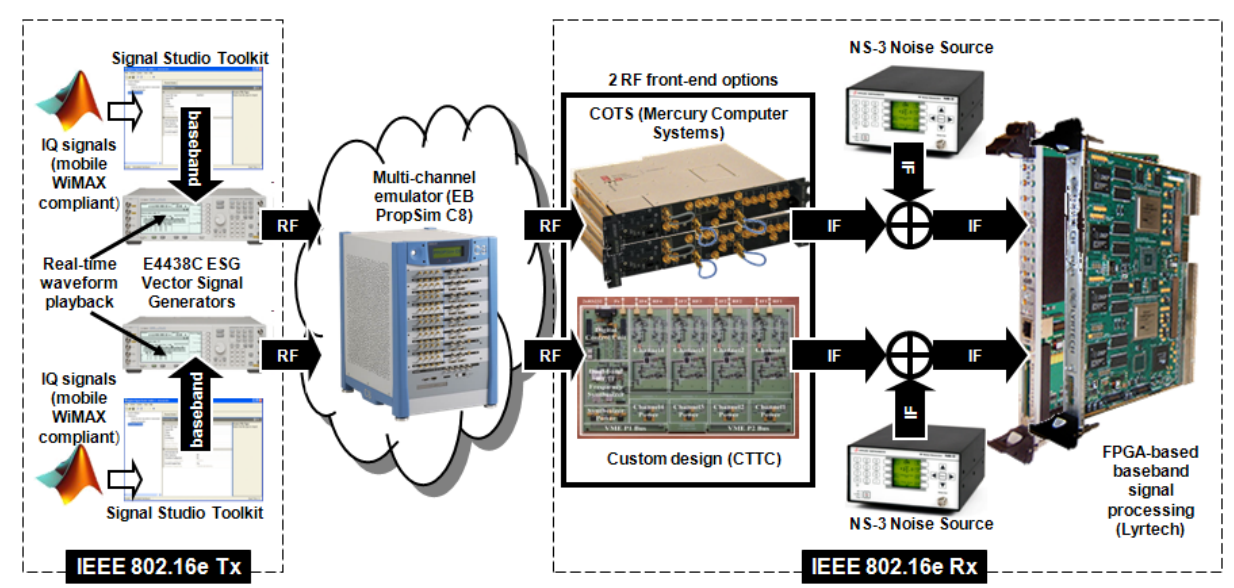

Figure 1: CTTC's point-to-point real time 2x2 MIMO testbed featuring mobile WiMAX.

Table 1: A synopsis of system parameters.

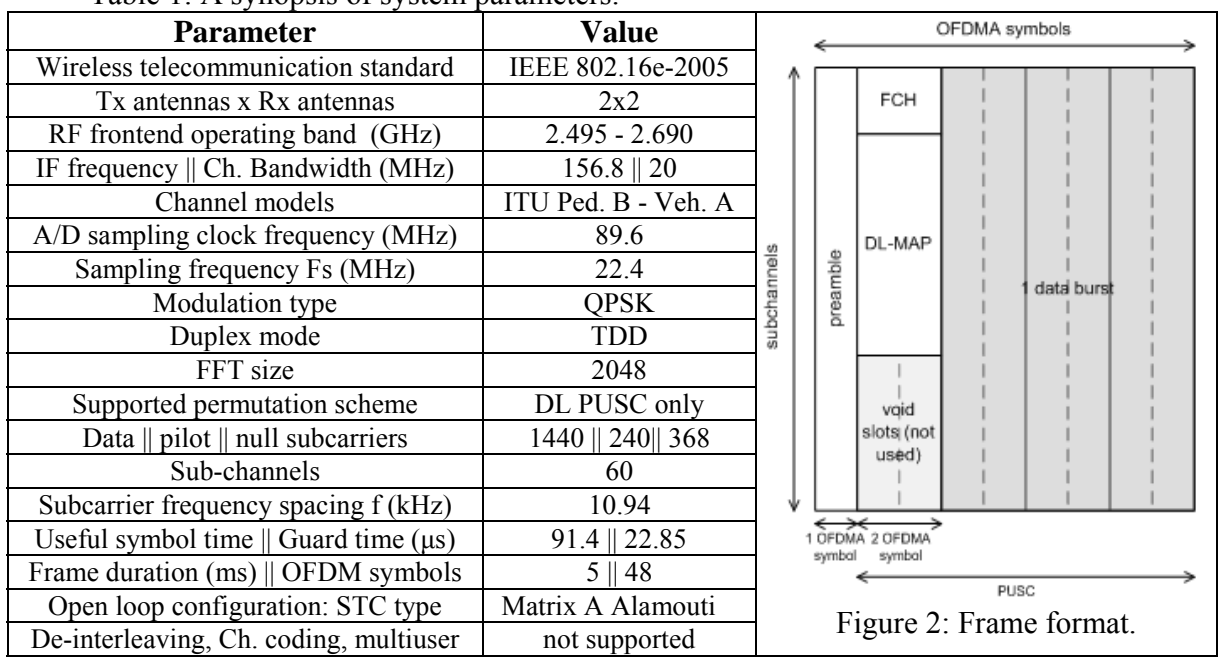

MIMO Signal Transmission: The baseband part of the transmitter was designed using Matlab. The separate I and Q baseband outputs of this model (corresponding to the two transmitter's branches) are written to data-files, which are fed to two instances of Agilent's Signal Studio Toolkit. The data-files are then uploaded to two Agilent vector signal generators (i.e. ESG4438C), which are appropriately connected for a MIMO signal transmission. This connection requires careful offline adjustments (e.g. time-alignment of the output signals). The two ESG4438C are utilizing their embedded arbitrary waveform generator to playback in real-time the baseband I and Q waveforms, up-convert the signal and finally provide the RF output centered at 2.595 GHz. The accuracy of these instruments guarantees a very high performance (e.g. excellent Error Vector Magnitude (EVM) profile). To verify the transmitter's 
compliance with the IEEE 802.16e standard, we have used Agilent's Vector Signal Analyzer (VSA) to demodulate the received RF signal.

The Channel: The connection between the transmitter and receiver can be done via a direct cable, over the free radio channel using antennas, or through a channel emulator. The two testing and measuring scenarios were: i) antenna transmission using an indoor radio channel, and ii) use of a channel emulator (i.e. EB Propsim C8) to generate an outdoor static or mobile channel. Measurements over the indoor radio channel were conducted only to prove functional conformity and thus they would not be analyzed herein. The channel emulator is configured with a 2x2 MIMO model, emulating the ITU Vehicular-A standard channel model (i.e. 6 tap tap-delay-line). The channel is assumed to be quasi static for the duration of an OFDM frame.

The Receiver: A fully integrated, dual-band multi-channel WiFi RF transceiver [10] (i.e. designed in CTTC) was upgraded to match the WiMAX testing scenario. Certain critical building blocks were replaced (i.e. RF and IF filters, the local oscillator and the sampling frequency synthesizer). Both the WiFi operation at 2.4 $\mathrm{GHz}$ and the WiMAX one at $2.6 \mathrm{GHz}$ performed satisfactory in a $2 \times 2$ MIMO configuration (i.e. proof of concept validation). The testbed specifications were expanded in terms of scalability and performance by acquiring high-end, multichannel broadband RF downconverters (i.e. MCS Echotek Series RF 3000T). Table 2 summarizes the main specifications of the two available RF front-end solutions.

Table 2: Performance-comparison of the RF front-end solutions.

\begin{tabular}{|c|c|c|}
\hline Parameter & Custom receiver & COTS receiver \\
\hline RF input frequency range & $2.4-2.7 \mathrm{GHz} \& 5.15-5.35 \mathrm{GHz}$ & $20 \mathrm{MHz}-3 \mathrm{GHz}$ \\
\hline IF output frequency range (3dB BW) & $135 . .173 \mathrm{MHz}$ & $107.5 . .172 .5 \mathrm{MHz}$ \\
\hline Frequency resolution & $<150 \mathrm{~Hz}$ & $1 \mathrm{~Hz}$ \\
\hline Internal reference accuracy & $< \pm 1 \mathrm{ppm}$ adj. & $< \pm 0.5 \mathrm{ppm}$ \\
\hline Phase Noise & $-83 \mathrm{dBc} / \mathrm{Hz} @ 10 \mathrm{KHz}$ & $-112 \mathrm{dBc} / \mathrm{Hz} @ 10 \mathrm{KHz}$ \\
\hline Noise Figure & $9.5 \mathrm{~dB}$ & $8.25 \mathrm{~dB}$ \\
\hline Gain control range & $72 \mathrm{~dB}$ & $85 \mathrm{~dB}$ \\
\hline Image rejection & $30 \mathrm{~dB}$ & $95 \mathrm{~dB}$ \\
\hline Spurious output levels & $-30 \mathrm{dBc}(\mathrm{LO}$ not inc.) & $-85 \mathrm{dBc}$ \\
\hline Input Third-Order Intercept Point (IIP3) & $-15 \mathrm{dBm}$ & $0 \mathrm{dBm}$ \\
\hline
\end{tabular}

The receiver also includes two noise signal generators (i.e. Applied Instruments NS-3) for testing purposes and a powerful baseband signal processing platform from Lyrtech Inc., which is assembled in a cPCI chassis. This includes an ADC board (i.e. 8 channels, 105 MSPS, 14-bit resolution, Xilinx Virtex-4 LX160 FPGA device), a signal processing board with 4 Xilinx Virtex-4 devices (i.e. 2 LX160 and 2 SX35) and 4 Texas Instruments TMS320C6416 DSPs. The platform also offers various I/O connectivity options (e.g. 8 Gbps data transfer between boards).

\section{Signal Model and Impairments}

The WiMAX signal is frame-based, composed of data and silence periods. The receiver is continuously monitoring the signal during the silence periods through a synchronization algorithm to detect the beginning of a data period. In real systems, on 
top of the noise, some system-wide signal-impairments appear during the silence due to the instrumentation used (e.g. signal generators, channel emulator). These "parasitic" signals impair the operation and performance of the system. Each one of these has been studied in order to remove its undesirable effects. The specifications of the signal generators, the channel emulator and RF downconverters, allow us to ignore the impact of the following signal-impairments: i) I/Q gain and phase imbalances due to variations in components between the analog I and Q processing branches, ii) inaccuracy between the sampling clocks of the transmitter and receiver, iii) random phase noise due to oscillator instability. Thus, the resulting received signal model at the output of the RF downconverters at the $i$ th antenna can be expressed as:

$$
r_{i}(t)=\operatorname{Re}\left\{x_{i}^{(R)}(t) \cdot e^{j 2 \pi\left(f_{I F}+\Delta f\right) t}\right\}+A_{i}+B_{i} \cos \left(2 \pi\left(f_{I F}+\Delta f\right) t+\varphi_{i}\right)+w_{i}(t),
$$

where $x_{i}^{(R)}(t)$ represents the useful part of the received baseband signal, $f_{I F}$ is the intermediate frequency (IF), $\Delta f$ is the carrier frequency offset (CFO), $A_{i}$ represents the DC level introduced by the baseband boards, $B_{i} \cos \left(2 \pi\left(f_{I F}+\Delta f\right) t+\varphi_{i}\right)$ represents the carrier located at the center of the useful signal-spectrum as a result of the coupling of the local oscillators at the transmitter and/or the receiver, and $w_{i}(t)$ is the Gaussian noise. In our case, the IF is $156.8 \mathrm{MHz}$ and the sampling frequency is $89.6 \mathrm{MHz}$ (oversampling by a factor of 4). This means that after the ADCs, one of the aliases of the discrete signal will be located at $22.4 \mathrm{MHz}$. The $x_{i}^{(R)}(t)$ can be expressed as:

$$
x_{i}^{(R)}(t)=\sum_{p=1}^{n_{T}} x_{p}^{(T)}(t) * h_{i, p}(t),
$$

where $x_{p}^{(T)}(t)$ is the equivalent baseband signal transmitted from the $p$ th transmit antenna and $h_{i, p}(t)$ is the equivalent baseband of the time impulse response of the MIMO channel between the $p$ th transmit and the $i$ th receive antennas. The total number of transmit antennas is assumed to be $n_{T}$.

Several countermeasures were employed to compensate as much as possible the effects of the received noise and some undesired spurious signals introduced by the channel emulator (e.g. prototyping of additional IF SAW filters).

\section{The Mobile WiMAX Receiver}

The digital front-end is one of the most critical processing stages of the receiver with determinant contribution to system's performance, highly susceptible to signal impairments and thus error-prone. It comprises from the automatic gain control (AGC), the digital down converter (DDC) and the synchronization block (figure 3). Any deviation from the expected signal-specification may either render the system's output invalid or seriously compromise its performance.

The AGC block adjusts the gain of the programmable gain amplifier to fit the dynamic range of the signal to the operating range of the ADCs preventing their saturation (excluding a back-off margin accounting for the OFDM signal crest factor) preventing their saturation and minimizing quantization errors. The DDC extracts the in-phase and quadrature components of the signal. This is achieved by a numerically 
controlled oscillator ( $\mathrm{NCO}$ ), initially tuned at the nominal frequency of $22.4 \mathrm{MHz}$, a digital mixer and a low-pass filter with a decimation factor of 4 (i.e. output baseband sampling frequency of $22.4 \mathrm{MHz}$ ). The filter was specifically designed to reject the DC level introduced before the ADCs by the baseband board chassis.

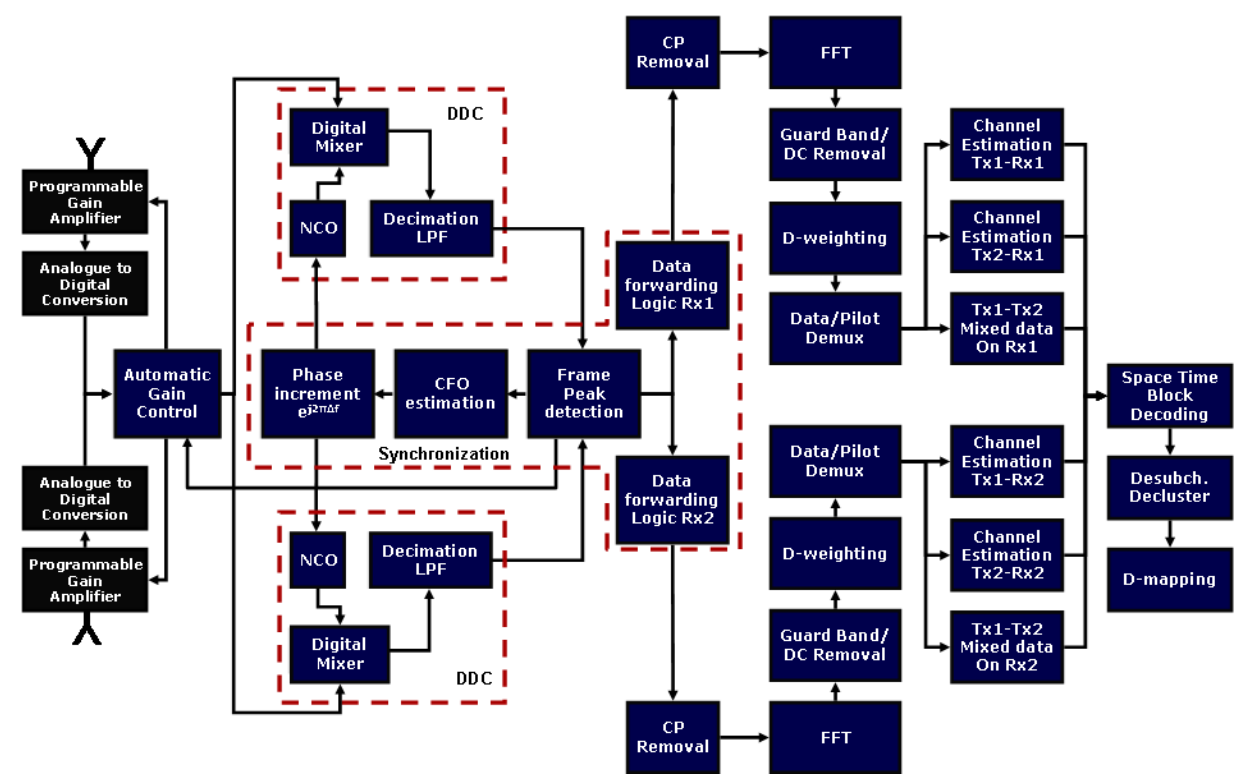

Figure 3: Overview of the processing components of the receiver.

The synchronization block has two main functions. First, it detects the beginning of the data period and, accordingly, the position of the OFDM symbols to apply the FFT. Second, it has to estimate any residual CFO that will be used to finely tune on-the-fly the NCO of the DDC. Both functions can be implemented jointly by calculating the cross-correlation of the received samples. Each OFDM symbol is composed of a Cycle Prefix (CP) of 512 samples and a useful part of 2048 samples. Since the CP samples copy that last samples of the useful part, it is expected that a peak in the modulus of the cross-correlation $r[n]$ between both sets of samples will appear if the position of the correlation window is correct. An additional mechanism will also prevent the false peak detection due to the presence of the carrier from the coupling of the local oscillators during the silence periods. In addition, the phase of the crosscorrelation at the peak will allow calculating the CFO. This can be mathematically expressed as follows (where $n_{R}$ denotes the number of receive antennas):

$$
\begin{gathered}
r[n]=\frac{\sum_{i=1}^{n_{R}} \sum_{k=0}^{511} x_{i}^{*}[n+k] \cdot x_{i}[n+k+2048]}{\sqrt{\sum_{i=1}^{n_{R}} \sum_{k=0}^{511}\left|x_{i}[n+k]\right|^{2}} \sqrt{\sum_{i=1}^{n_{R}} \sum_{k=0}^{511}\left|x_{i}[n+k+2048]\right|^{2}}}, \\
n_{\max }=\arg \max _{n}|r[n]|^{2}, \quad \Delta f=\frac{1}{2 \pi} \frac{22.4 \cdot 10^{6}}{2048} \cdot \arg \left\{r\left[n_{\max }\right]\right\}
\end{gathered}
$$


In the following processing stages, the OFDM demodulation takes place; this includes the CP removal, the FFT, and the removal of the guard band and the DC carriers. The subsequent blocks are related with the organization, randomization and grouping of the carriers according to the IEEE 802.16e frame definition, and the pilots' extraction which are scattered along the signal bandwidth (where these pilots are used to estimate the channel response). When the system is configured as a MIMO one, each processing-chain at the receiver has to estimate the corresponding channels from all transmit antennas. This is carried out by extracting the channel frequency response at the pilots' positions and then interpolating them by using a second order polynomial interpolation as indicated in formula (4).

$$
f(x)=y_{p 1}+\frac{\left(y_{p 2}-y_{p 1}\right)}{\left(x_{p 2}-x_{p 1}\right)} \cdot\left(x-x_{p 1}\right)+\frac{\left(y_{p 3}-y_{p 2}\right)}{\left(x_{p 3}-x_{p 2}\right)}-\frac{\left(y_{p 2}-y_{p 1}\right)}{\left(x_{p 2}-x_{p 1}\right)} \cdot\left(x-x_{p 1}\right) \cdot\left(x-x_{p 2}\right)
$$

One of the final blocks is related with the decoding of Alamouti's code (matrix A), which is applied on a per-subcarrier basis in 2-transmit antenna systems. The two equations (5) show the operation to be applied for estimating the transmitted symbols at the $k$ th subcarrier. Two consecutive OFDM symbols, $2 l$ and $2 l+1$, have to be processed jointly, where the samples at each antenna of the receiver after the FFT are represented by $R_{i}$ and the estimated channel frequency response is denoted by $H_{i, p}$ :

$$
\hat{S}_{N}=\frac{\sum_{i=1}^{n_{R}} H_{i, 1}^{*}[k] \cdot R_{i}[k, 2 l]+H_{i, 2}[k] \cdot R_{i}^{*}[k, 2 l+1]}{\sum_{i=1}^{n_{R}}\left|H_{i, 1}[k]\right|^{2}+\left|H_{i, 2}[k]\right|^{2}}, \quad \hat{S}_{N+1}=\frac{\sum_{i=1}^{n_{R}} H_{i, 2}^{*}[k] \cdot R_{i}[k, 2 l]-H_{i, 1}[k] \cdot R_{i}^{*}[k, 2 l+1]}{\sum_{i=1}^{n_{R}}\left|H_{i, 1}[k]\right|^{2}+\left|H_{i, 2}[k]\right|^{2}}
$$

The design has passed through numerous optimization stages in order to boost the performance and minimize the processing complexity (i.e. FPGA slices, RAMB16s utilization). The $2 \times 2$ MIMO receiver was fitted in two Virtex-4 LX160 devices, using the Xilinx ISE 9.2 design-suite (FPGA1: 81\% slices, 93\% RAMB16s, 100\% DSP48 and FPGA2: 49\% slices, 71\% RAMB16s, 57\% DSP48). The compilation time of the MIMO configuration reached a peak aggregate of 40 hours in a dedicated 64-bit server. The dense device utilization resulted in a volatile implementation, since the place and routing process only manages to meet timing constraints in an arbitrary way. This limitation is posed by the ISE software and the only solution is to apply more stringent timing constraints and divide the design targeting 3 FPGA devices.

\section{Results and Conclusions}

The data at the end of the processing chain of the mobile WiMAX receiver is captured and visualized in real-time with the help of the Chipscope Pro software from Xilinx. This software generates monitoring cores which are attached to the target design running in the FPGAs of the testbed. The accuracy of the received constellation points is investigated at the receiver by calculating the EVM, which is a metric of their dispersion from the ideal positions [11]. To compare the performance 
of the implementation we have also captured and stored in a data file a real signal in the output of the ADCs which we have then fed to the respective Matlab model of the receiver. Thus, by comparing both results, we can effectively measure which is the degradation in the system performance due to the numerical approximations (finite bit precision) performed by the hardware implementation of the testbed.

An indicative comparison of how the QPSK constellation of the received signal is visualized using Matlab on the one hand and on-board real-time data-capturing on the other, is shown in figures 4, 5 (i.e., $\mathrm{SNR}=15.34 \mathrm{~dB}$ ) and figures 6, 7 (i.e. $\mathrm{SNR}=$ $21.69 \mathrm{~dB})$ respectively. The SNR represents the function of the total signal power over the total noise power across the bandwidth of the received signal.

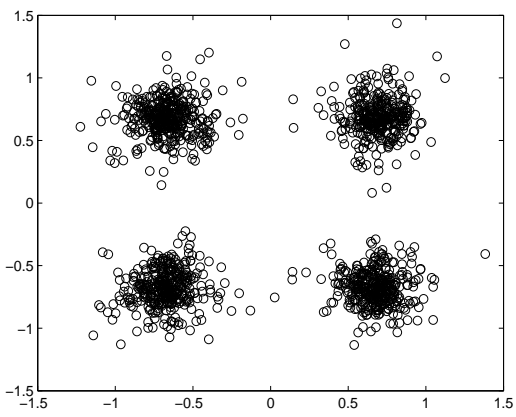

Figure 4: Matlab-SNR=15.34dB.

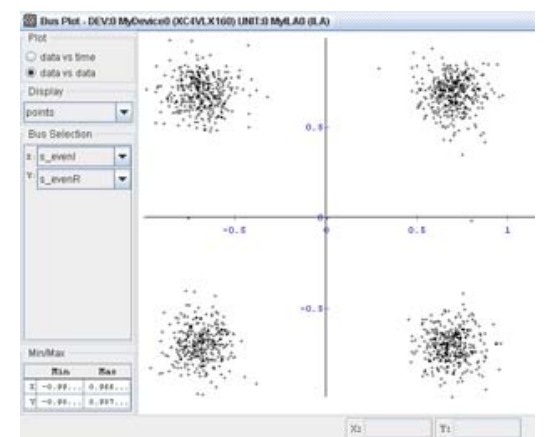

Figure 5: On-board-SNR=15.34dB

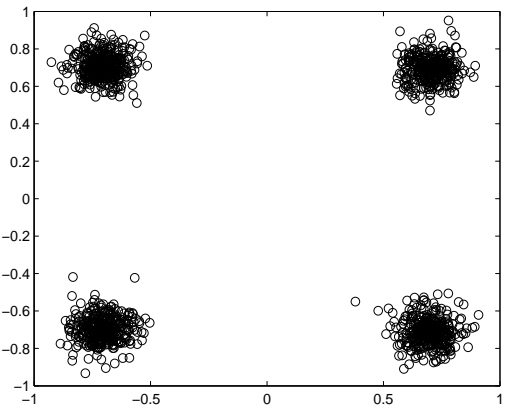

Figure 6: Matlab-SNR=21.69dB.

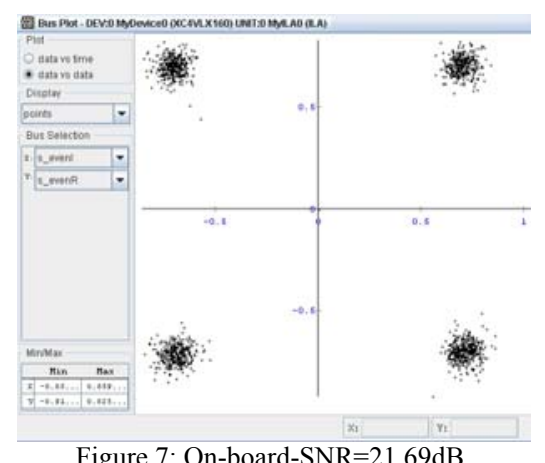

Figure 7: On-board-SNR=21.69dB.

The two previous figures show a capture of the received QPSK constellations at two indicative SNR values. In order to further evaluate the precision and performance of the implemented system, more detailed numerical results have been obtained. These correspond not only to the aforementioned EVM, but also to the raw BER (i.e., assuming no channel coding) for both the Matlab model (i.e., "ideal" receiver) and the actual hardware implementation of the receiver. Note that the EVM is approximately calculated since the deviations of the received constellation points are measured with respect to the taken QPSK de-mapping decisions, instead of the actual ideal error-free constellation points. In this sense, this approximate EVM will be more accurate at high SNR, i.e., when less errors occur in the decisions. The results are obtained for a fixed channel and for different values of the received SNR by adjusting the 


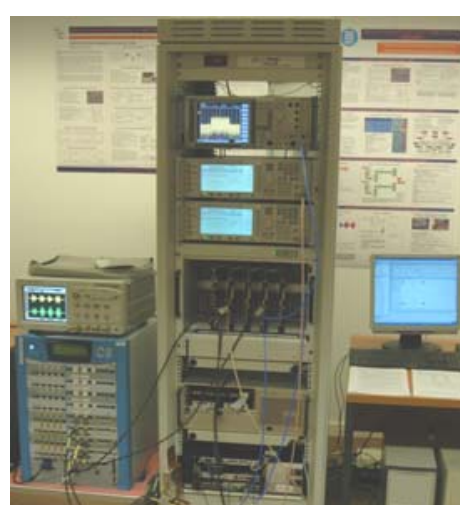

Figure 8: CTTC real-time testbed. Left: channel emulator, oscilloscope. Rack: (top to

bottom) spectrum analyzer, signal generators, RF frontends, baseband boards. attenuation of two noise generators. The EVM and BER curves, which are shown in figures 9 and 10, demonstrate the robustness and precision under implementation losses within an acceptable margin.

The deviation observed in the raw BER curves is due to certain approximations made in the processing chain at the HW receiver, which ultimately affected the EVM calculation; the loss of precision due to the fixed-point implementation of critical building blocks of the system (e.g. channel estimation) deteriorate the constellation on top of the impairment introduced by the observed SNR. In high SNR scenarios, this fixed-point arithmetic-conversion deterioration will only affect few points in the constellation, thus, increasing the raw BER but not equally the approximate EVM, as the QPSK d-mapping decision could be wrong.

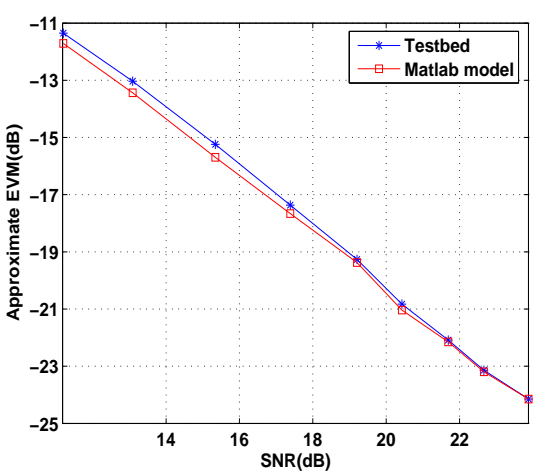

Figure 9: Performance comparison between the Matlab model and the real-time testbed, through the approximate EVM-SNR curves.

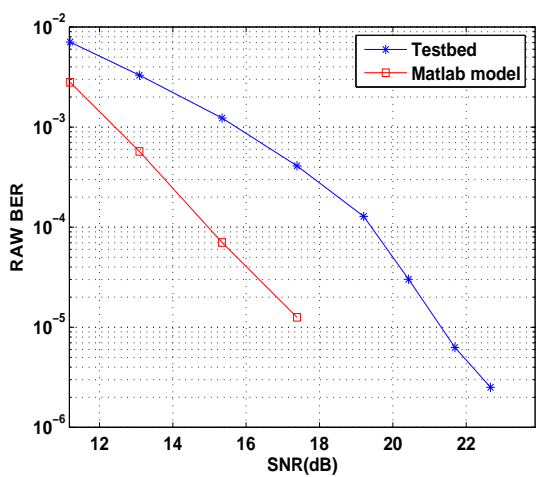

Figure 10: performance comparison between the Matlab model and the real-time testbed, through the raw BER-SNR curves.

This paper presented a real-time MIMO testbed featuring a mobile WiMAX system. This is rightfully considered a challenging task requiring a resourceful budget, manpower, time and hands-on expertise on advanced signal processing aspects. The testbed comprises the necessary technology, equipment and specifications that allow the implementation of top performance systems. The experimental setup of the $2 \times 2$ MIMO testbed operating in CTTC is shown in figure 8 . The deployment of an IEEE 802.16e-2005 2x2 MIMO real-time system operating beyond the typical WiMAX specifications (i.e. wave-2), with a $20 \mathrm{MHz}$ channel bandwidth is giving us the opportunity to test and experiment state-of-the-art research concepts. 
The next development step will include the implementation of a real-time transmitter replacing the signal generators with a custom FPGA development. This will open several new research and implementation possibilities, such as the inclusion of more advanced MIMO exploitation schemes based on real-time feedback. This feedback from the receiver to the transmitter could deliver information about the current channel conditions and would allow the transmitter to adapt its transmission scheme to such channel, thus, boosting the system performance and making it more efficient. Some schemes to be analyzed are based on antenna selection or adaptive beamforming based on codebooks. Moreover, the feedback will enable us to explore more advanced configurations, such as multi-user networks based on opportunistic transmission, e.g., allocating carriers to users in a dynamic way.

\section{References}

1. Mobile WiMAX air interface, P802.16Rev2/D9, (Revision of IEEE Std 802.16-2004 and consolidates material from IEEE Std 802.16e-2005, IEEE Std 802.16-2004/Cor1-2005.

2. Kuhling, D., Ibing, A., Jungnickel, V.: 12×12 MIMO-OFDM realtime implementation for 3GPP LTE+ on a Cell Processor. In: 14th European Wireless Conference, pp. 1-5. Prague, Czech Republic, 22-25 June 2008.

3. Alamouti, S.: A simple transmit diversity technique for wireless communications. in IEEE Journal on Selected Areas in Communications, vol. 45, no. 9, pp. 1451-1458, 1998.

4. Ramirez, D., Santamaria, I., Perez, J., Via, J., Tazon, A., Garcia-Naya, J.A., FernandezCarames, T.M., Gonzalez Lopez, M., Perez-Iglesias, H., Castedo, L.: A Flexible Testbed for the Rapid Prototyping of MIMO Baseband Modules. In: 3rd International Symposium on Wireless Communication Systems, pp. 776-780. Valencia, Spain, 6-8 Sept. 2006.

5. Su Hu, Gang Wu, Yong Liang Guan, Choi Look Law, Yanxin Yan, Shaoqian Li: Development and performance evaluation of mobile WiMAX testbed. In: IEEE Mobile WiMAX Symposium, pp. 104-107. Orlando, USA 25-29 March 2007.

6. Haene, S., Perels, D., Burg, A.: A Real-Time 4-Stream MIMO-OFDM Transceiver: System Design, FPGA Implementation, and Characterization. In: IEEE Journal on Selected Areas in Communications, Aug. 2008, Vol. 26, Issue 6, pp: 877-889.

7. Ramirez, D., Santamaria, I., Perez, J., Via, J., Garcia, J.A., Fernandez, T., Perez, H. J., Gonzalez, M., Castedo, L., Torres, J.M.: A comparative sutdy of STBC transmissions at $2.4 \mathrm{GHz}$ over indoor channels using a 2x2 MIMO tested. In Wireless Communications and Mobile Computing, Vol. 8, Nov. 2008, pp. 1149-1164.

8. Víctor P. Gil Jiménez, M. Julia Fernández-Getino García, Ana García Armada, et al.: A MIMO-OFDM Testbed, Channel Measurements, and System Considerations for OutdoorIndoor WiMAX. In EURASIP Journal on Wireless Communications and Networking, vol. 2010, Article ID 871291, 13 pages, 2010.

9. Mignanti S., Castellano M., Spada M., Simoes P., Tamea G. et al.: WEIRD Testbeds with Fixed and Mobile WiMAX Technology for User Applications, Telemedicine and Monitoring of Impervious Areas. In proceedings of TridentCom 2008, Innsbruck, Austria, March 18-20, 2008.

10. Nieto, X., Ventura, L., Mollfulleda, A.: GEDOMIS: A Broadband Wireless MIMOOFDM Testbed. Design and Implementation. In Proceedings of. TridentCom 2006, pp. 121-131. Barcelona-Spain, March 1-3 2006.

11. Shafik, R. A., Rahman, M. S. and Islam, A. H. M. R.: On the Extended Relationships Among EVM, BER and SNR as Performance Metrics. In 4th International Conference on Electrical and Computer Engineering, pp. 408-411. Dhaka, Bangladesh December, 2006. 